\title{
Method for assessment of matching of total ankle joint endoprostheses based on CT data
}

\begin{abstract}
The investigation of matching of endoprosthesis tibial components to the bone cross section is of interest for the manufacturer as well as for the surgeon. On the one hand, a systemic design of the prosthesis and the assortment is possible, on the other hand, a better matching implantation is enabled on the basis of experience of this study.

CT sections were segmented manually using a CAD system and fitted by spline functions, then superseded with cross sections of the tibial component of a modified Hintermann $\mathrm{H} 3$ prosthesis.

The principal moments of inertia, the direction of the principal axes and the area of the section were evaluated. Based on the relative differences of the principal moments of inertia, recommendations for application of the different prosthesis size and its selection with the surgery can be made.
\end{abstract}

Keywords: Traumatology, biomechanics, total ankle prosthesis, fixation

https://doi.org/10.1515/cdbme-2020-3102

\section{Introduction}

The application of current total ankle joint prostheses requires a systematic investigation of tibial component matching to bone section geometry based on CT data.

With the Hintermann $\mathrm{H} 3$ prosthesis [1] this can be achieved by matching different prosthesis sizes to CT sections. An investigation of matching accuracy is required to obtain a good matching of prosthesis to bone as well as to keep the number of prostheses sizes as well as the costs

*Corresponding author: Heiner Martin: University medicine Rostock, Institute for Biomedical Engineering, F.- Barnewitz-Str. 4, 18119 Rostock, e-mail: heiner.martin@uni-rostock.de

Josephine Wittmüß, Thomas MittImeier: University medicine Rostock, Dept. of trauma, hand and reconstructive surgery, 18057 Rostock, Schillingallee 35

Niels Grabow: University medicine Rostock, Institute for Biomedical Engineering, F.- Barnewitz-Str. 4, 18119 Rostock within a reasonable limit.

With a significant difference of prosthesis component section compared with the bone section, instability of prosthesis components is to be expected. Since a good match will result with good ingrowth behaviour to a high bending stiffness of the bone-prosthesis combination, the principal moments of inertia and the principal axis orientation were investigated as an indicator for the prosthesis match. Moreover, the demand for the different prosthesis sizes had to be balanced, and the relative difference of minimum and maximum moments of inertia had to be investigated.

\section{Material and method}

Using 436 CT data sets of tibia cross sections, different characteristic values of the implantations were examined:

- the relative differences of maximum and minimum moment of inertia

- the frequency of usage of different prosthesis sizes

- the cross section area

- the angle between principal axis and axis of CT coordinate system.

Especially, a low relative difference between the principal moments of inertia is characteristic for approximately circular or quadratic cross sections.

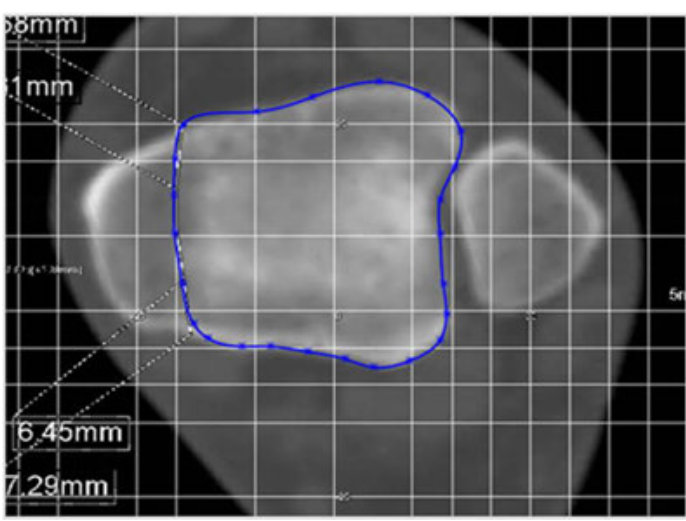

Figure 1: Cross section of tibia in resection plane with approximation by splines 


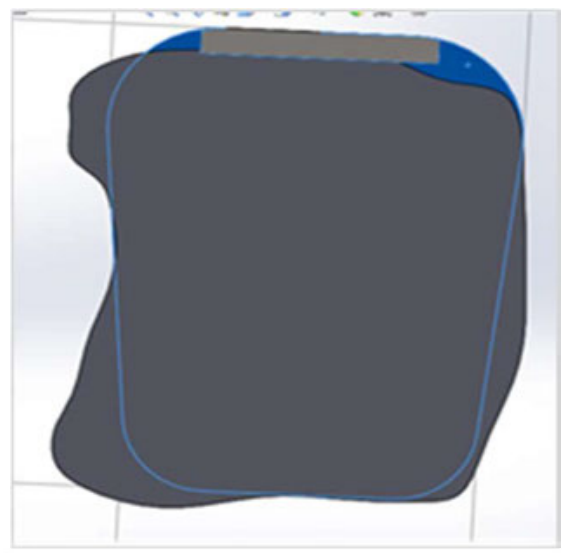

Figure 2: Exemplary superseding of a bone cross section with a prosthesis component

CT images of the bone cross section in the resection plane were imported into a CAD system and segmented manually (Fig. 1). The cross section contour was fitted by spline functions and manually superimposed by the given tibial component section.

The contact area as well as the principal axis and moments of inertia could be determined thereby (Fig. 2).

Under the assumption of an equally good contact between bone and prosthesis surface, the contact area is essential for the implant ingrowth and thereby for the combination of bone and prosthesis.

\section{Results and discussion}

The following essential results were found:

The cross section area results show an approximately symmetric distribution around an average of about $1000 \mathrm{~mm}^{2}$. The given five prosthesis sizes covered the whole area range.

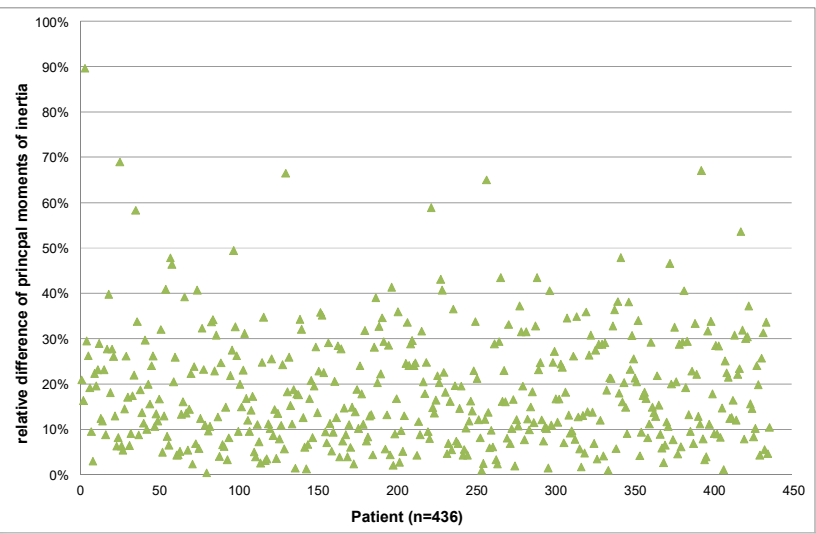

Figure 3: Relative differences of principal moments of inertia of all patients

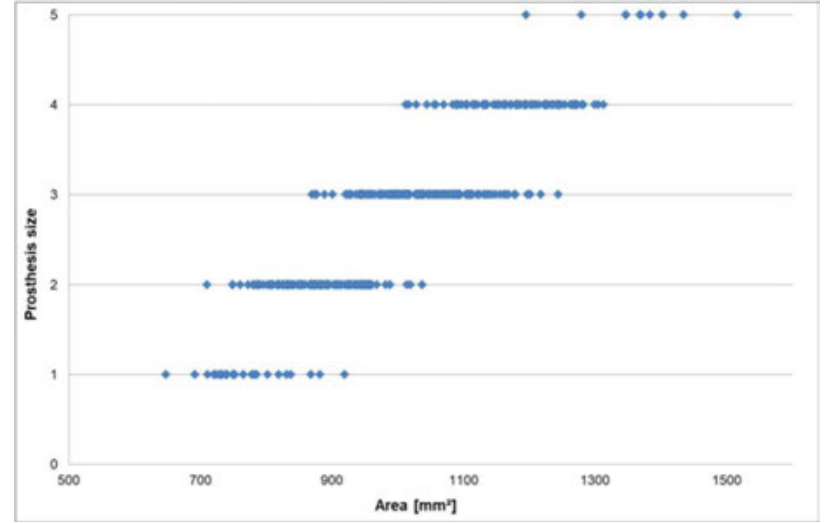

Figure 4: Diagram displaying the assignment of cross section area to prosthesis size

Corresponding to the frequency distribution the middle prosthesis sizes are appropriate in the most cases (Fig. 3).

The relative difference of the principal moments of inertia was less than $40 \%$ in $95 \%$ of all implantations (Fig. 4). Hence, in the most cases quadratic or circular contact areas are to be expected.

The initial hypothesis that the angle of the principal axes to the CT coordinate system would be in the most cases approximately $0^{\circ}$ or $90^{\circ}$ could not be confirmed due to the accidental position of the most patients in CT.

\section{Conclusion}

Concluding it can be stated that the geometric evaluation of tibial cross section area together with the prosthesis cross section can give valuable information on a better design of the tibial component as well as for stability and ingrowth behaviour. Furtheron, a relevant hint is given with respect to the corresponding size distribution.

\section{Author Statement}

Research funding: The author state no funding involved. Conflict of interest: Authors state no conflict of interest. Informed consent: Informed consent has been obtained from all individuals included in this study. Ethical approval: The research related to human use complies with all the relevant national regulations, institutional policies and was performed in accordance with the tenets of the Helsinki Declaration, and has been approved by the authors' institutional review board or equivalent committee.

\section{References \\ [1] Beat Hintermann: Endoprothetik des Sprunggelenks. Springer Wien New York 2005.}

